\title{
Absorption of Acoustic Waves in Dilute Solutions and Dilute Suspensions of Polymers at Frequencies 10 to $300 \mathrm{kHz}$
}

\author{
Toshihiko OHSAWA* and Yasaku WADA \\ Department of Applied Physics, Faculty of Engineering, \\ University of Tokyo, Bunkyo-ku, Tokyo, Japan.
}

(Received April 28, 1970)

\begin{abstract}
Acoustic absorption of dilute solutions of polystyrene in xylene is measured at frequencies 10 to $300 \mathrm{kHz}$ by means of the resonance reverberation method. Dynamic volume viscosity as a function of frequency is obtained for solutions of polystyrene of three different molecular weights. The data exhibits relaxational behavior in the covered frequencies. The relaxation has a much higher relaxation strength than shear relaxation predicted by the Rouse theory. The distribution of relaxation times is broad and seems to join with the acoustic relaxation at megahertz range which has been attributed by Hässler and Bauer to crank-shaft motion of polymer segments. Acoustic absorption of dilute aqueous suspensions of styrene-butadiene rubber is measured and the imaginary part of complex bulk modulus of the rubber is calculated. The loss modulus increases with increasing frequency, suggesting the loss peak due to the primary relaxation is positioned at a higher frequency.

KEY WORDS Relaxation/Ultrasonics / Polystyrene/Dilute Solutions/ Suspension / SBR / Reverberation Method /
\end{abstract}

The improved resonance reverberation method described in previous papers ${ }^{1,2}$ has proved to be an effective tool for measuring sound velocity and absorption in a liquid at frequencies from 10 to $300 \mathrm{kHz}$. The purpose of this study is to show examples of application of this method to polymeric systems.

Viscoelasticity of dilute solutions of flexible chain polymers in shear deformation has been widely studied from both theoretical and experimental points of view. ${ }^{3}$ Results indicate the theories by Rouse ${ }^{4}$ and $\mathrm{Zimm}^{5}$ based on the submolecule model can fairly well interpret the experimental data at low frequencies. On the other hand, study of viscoelasticity of dilute polymer solutions in volume deformation is limited to the megahertz range where the conventional pulse technique is applied. Since the shear relaxation is predominant below $100 \mathrm{kHz}$ for usual temperatures and solvents, it may be interesting Ltd.

On leave from Japan Synthetic Rubber Co.,

Present address: Department of Applied Physics, Faculty of Technology, Tokyo University of Agriculture and Technology, Koganei, Tokyo, Japan. to measure the acoustic relaxation at these frequencies. In the first part of the present study, the absorption coefficient of dilute polystyrene solutions in xylene is measured varying frequency by means of the resonance reverberation method.

The second part of this study is devoted to acoustic absorption in dilute suspensions of polymers. The absorption of acoustic waves in suspensions and emulsions generally originates from four sources: ${ }^{6,7}$ (1) $\alpha_{\mathrm{s}}$, scattering of sound waves, (2) $\alpha_{v}$, the viscous loss at the interface of particles and medium, (3) $\alpha_{t}$, loss due to thermal conduction between particles and medium, and (4) $\alpha_{i}$, internal loss of suspended particles. Experiments on this problem have so far been limited to above $300 \mathrm{kHz}$ where the pulse technique was employed. In this paper, the absorption coefficient in aqueous suspensions of styrenebutadiene rubber (SBR) is measured at frequencies from 10 to $300 \mathrm{kHz}$ and the result is analysed by existing theories.

\section{EXPERIMENTAL}

The apparatus used in the present study was the same as fully described elsewhere. ${ }^{1,2}$ A 
spherical pyrex flask 9.7 litres in volume was used throughout this study. Xylene and water were used as a reference liquid for polystyrene solutions in xylene and aqueous suspensions of SBR, respectively.

Polystyrene of three different molecular weights was used, designated $\mathrm{A}, \mathrm{B}$, and $\mathrm{C}$ in the following. Sample B was a commercial polystyrene (Styron 683) and samples $A$ and $C$ were prepared by bulk-polymerization using benzoyl peroxide as an initiator. The molecular weight distribution obtained by the gel-permeation chromatography is illustrated in Figure 1. The average molecular weight was determined from the curves as $1.5 \times 10^{5}, 3.4 \times 10^{5}$, and $7.0 \times 10^{5}$ for $\mathrm{A}$, $\mathrm{B}$, and $\mathrm{C}$, respectively. The steady-flow shear viscosity of solutions in the concentration of $1 \mathrm{wt} \%$ was $1.23 \times 10^{-2}, 1.42 \times 10^{-2}$ and $3.00 \times$ $10^{-2}$ poises at $20^{\circ} \mathrm{C}$, for $\mathrm{A}, \mathrm{B}$, and $\mathrm{C}$, respectively.

The absorption coefficient of two kinds of

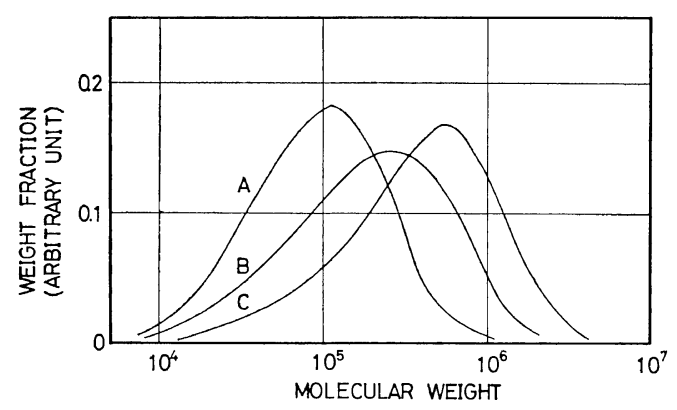

Figure 1. Molecular weight distribution of polystyrenes.

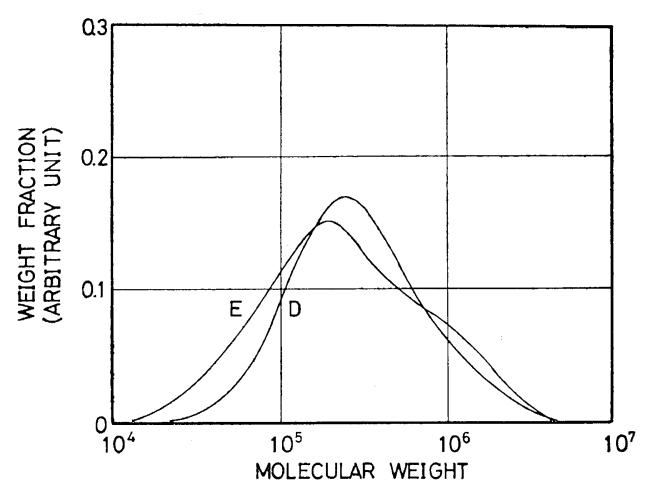

Figure 2. Molecular weight distribution of styrenebutadiene rubbers. suspensions of SBR (SBR 1500 and SBR 1712, designated $\mathrm{D}$ and $\mathrm{E}$, respectively) was measured by the resonance reverberation method, varying frequency and concentration. The styrene content was $23.5 \%$ in weight for both D and E. Suspension medium was water including $1.5 \%$ of surface reactant. The diameter of rubber particles obtained by the electron-micrograph was about $800 \AA$. The two samples had different molecular weights, as shown in Figure 2, where it can be seen that sample $E$ has a higher average molecular weight but includes a larger fraction of low molecular weight components.

\section{POLYSTYRENE SOLUTIONS}

Results for polystyrene solutions are shown in Figure 3, where the absorption coefficient per squared frequency is plotted against logarithmic

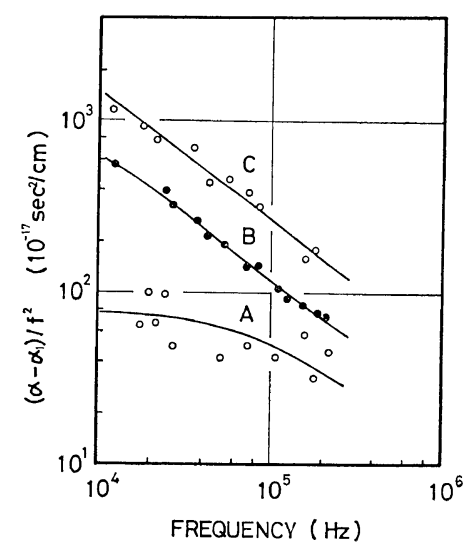

Figure 3. Absorption coefficient per squared frequency in dilute solutions of polystyrene in xylene at $20^{\circ} \mathrm{C}$ plotted against frequency: concentration, $1 \mathrm{~g} / 100 \mathrm{~g} ; \mathrm{mol} \mathrm{wt}, 1.5 \times 10^{5}, 3.4 \times 10^{5}$, and $7.0 \times 10^{5}$ for $\mathrm{A}, \mathrm{B}$, and $\mathrm{C}$, respectively.

frequency. The absorption coefficient $\alpha / f^{2}$ of a dilute solution is related in general to dynamic viscosities as

$$
\frac{\alpha-\alpha_{1}}{f^{2}} \simeq \frac{2 \pi^{2}}{\rho_{1} u_{1}^{3}}\left[\left(\kappa^{\prime}-\kappa_{1}\right)+\frac{4}{3}\left(\eta^{\prime}-\eta_{1}\right)\right]
$$

where $\rho$ is the density, $u$ the sound velocity, $\kappa^{\prime}$ the dynamic volume viscosity and $\eta^{\prime}$ the dynamic shear viscosity. The suffix 1 stands for 
the solvent. The viscosity $\left(\kappa^{\prime}+4 \eta^{\prime} / 3\right)$ calculated from $\left(\alpha-\alpha_{1}\right) / f^{2}$ using eq 1 is drawn in Figure 4, where broken lines represent $\eta^{\prime}$ estimated from the Rouse theory. ${ }^{4}$ The result in Figure 4 indicates that the absorption of acoustic waves is predominantly determined by the volume viscosity and the contribution from shear viscosity is negligible.

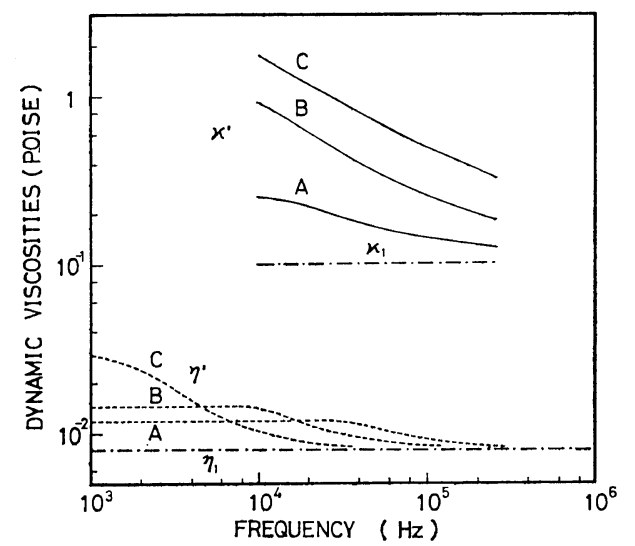

Figure 4. Dynamic viscosities of polystyrene solutions $\mathrm{A}, \mathrm{B}$, and $\mathrm{C}$ plotted against frequency. Dotted lines represent dynamic shear viscosity calculated from the Rouse theory.

Figure 3 reveals that the relaxational behavior contributes to dynamic volume viscosity in the covered frequency range and the value of $\kappa^{\prime}$ increases with increasing molecular weight. The result does not cover the overall behavior of the relaxation but the distribution of relaxation times may be very broad.

The theory of Okano, ${ }^{8}$ who treated the volume viscoelasticity of flexible chain polymers represented by the Rouse model, predicts that the relaxation times are the same for volume and shear deformations and the relaxation strength is also similar in order of magnitude. The result in the present study indicates the volume viscosity in dilute polymer solutions should have another origin, which is not taken into account in Okano's theory, developed under assumptions of the Rouse model and the isothermal process. In the adiabatic acoustic process, some kinds of thermal relaxation which are brought about by the reaction related to intramolecular vibration, internal rotation in molecules, and/or molec- ular rearrangement might occur in polymer solutions.

Recent acoustic studies ${ }^{9-11}$ on dilute solutions of polystyrene above $1 \mathrm{MHz}$ revealed the existence of a relaxation around $5 \mathrm{MHz}$. According to Hässler and Bauer, ${ }^{9}$ the relaxation may be attributed to the crank-shaft motion of a small segment which is the elementary process of micro-Brownian motion of polymers.

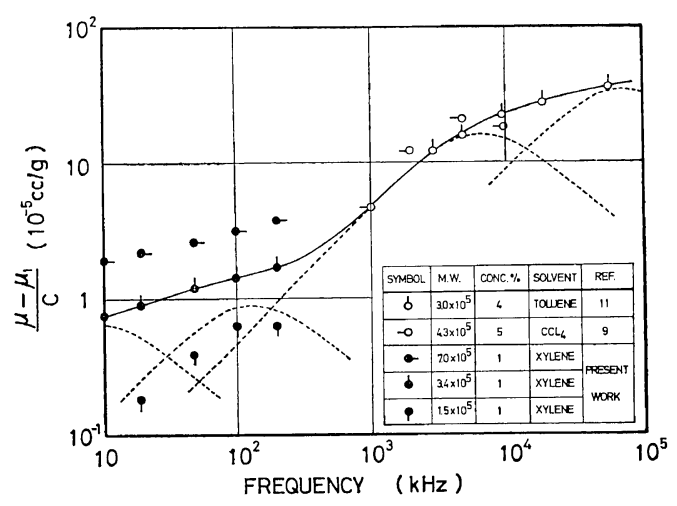

Figure 5. Excess absorption per wavelength reduced to unit polymer concentration in dilute solutions of polystyrene. Points represent smoothed values.

In Figure 5, the excess absorption per wavelength divided by the polymer concentration, $\left(\mu-\mu_{1}\right) / c$, is plotted against frequency in both logarithmic scales. At higher frequencies above $1 \mathrm{MHz}$, the excess absorption seems somewhat independent of molecular weight. The problem whether the excess absorption does drop above 5 megahertz or not depends largely on the choice of high-frequency limit, $\mu_{1}$. At lower frequencies below $1 \mathrm{MHz}$, on the other hand, the absorption clearly depends on molecular weight.

The solid line in Figure 5 represents values for sample B in the present study and those by Nomura, et al., ${ }^{11}$ in which a sample of a similar molecular weight and a solvent similar to the present study for sample B were used. The solid line in Figure 5 can be fitted by superposition of four relaxations, each being of single relaxation time, as illustrated by the dashed lines in Figure 5. Such treatment is, however, rather sophistic and it may be more appropriate to say that there exists a broad distribution of relaxation times in the entire range of frequency. 


\section{T. OHSAWA and Y. WADA}

Since a polymer has a large number of freedoms in internal rotation, a broad distribution of relaxation time is quite natural. The size of the segment involved with the crank-shaft motion may be small for short relaxation times but large for long ones. In the latter, the effect of the motion may extend to long range in a molecule and hence the absorption depends strongly on molecular weight.

Further work will be required to clarify this problem and will lead to an understanding of the nature of segmental or micro-Brownian motion of polymers.

It should be noted that solutions of polystyrene in benzene exhibit smaller acoustic absorption than solvent at megahertz range, ${ }^{9}$ similar to benzene including a small amount of low molecular weight impurity. The high absorption in benzene is attributed to thermal relaxation due to coupling of translational motion and intramolecular vibration. The decrease in absorption is interpreted by the shift of relaxation frequency to high due to acceleration of energy transfer through impurities.

The decrease in absorption in a polystyrene solution may probably come from the same mechanism and hence the thermal relaxation due to intramolecular vibration in polymers seems to contribute more or less to the acoustic absorption.

\section{SBR SUSPENSIONS}

Results for samples D and $E$ are represented in Figure 6, where the excess absorption $\left(\alpha-\alpha_{1}\right)$ is plotted against frequency for various concentrations. The result at a high concentration, $1.47 \%$, is not so reliable because of high decayrate. The two samples have different $\left(\alpha-\alpha_{1}\right)$ values when compared at the same frequency and the same concentration. Excess absorption per unit concentration $\left(\alpha-\alpha_{1}\right) / c$ is plotted in Figure 7.

As has been described in the first section of this paper, the excess absorption in suspensions $\left(\alpha-\alpha_{1}\right)$ is ascribed to four origins. The first of them, the scattering loss, is given ${ }^{12}$ under the condition $k a \ll 1$ by

$$
\alpha_{\mathrm{s}} / c=\frac{k}{2}\left[\frac{1}{3}(1-\tau)^{2}+\left(\frac{\sigma-1}{2 \sigma+1}\right)^{2}\right](k a)^{3},
$$

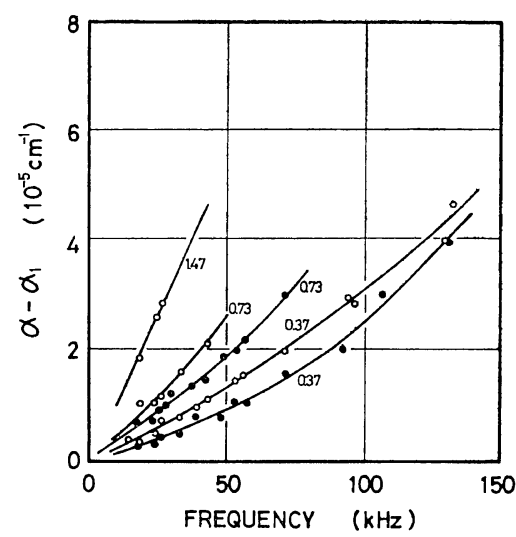

Figure 6. Absorption coefficient of aqueous suspensions of SBR, sample D (open circle) and sample $\mathrm{E}$ (filled circle) at different volume concentrations (\%) as indicated, $19^{\circ} \mathrm{C}$.

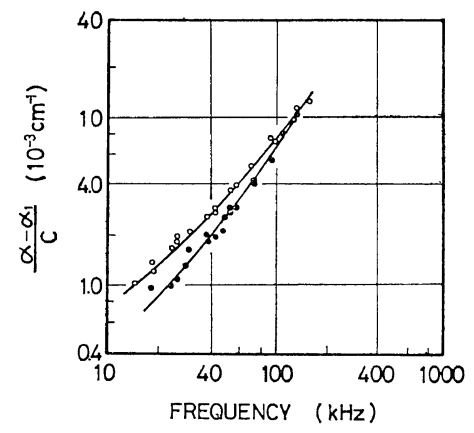

Figure 7. Excess absorption per unit concentration of SBR suspensions, sample D (open circle) and sample $\mathrm{E}$ (filled circle).

where $k$ is the propagation constant of acoustic waves, $k=2 \pi / \lambda=\omega / u$ ( $\lambda$, wavelength, $\omega$, angular frequency), $\sigma=\rho_{2} / \rho_{1}$ the density ratio, $\tau=K_{2}{ }^{\prime} / K_{1}{ }^{\prime}$ the ratio of bulk moduli, $a$ the particle radius, and $c$ the volume fraction of particles. Suffices 1 and 2 stand for medium and particle, respectively. In the present case, $k a \simeq$ $10^{-4}$ at $100 \mathrm{kHz}$. The value of $\alpha_{\mathrm{s}} / c$ estimated from eq 2 is in the order of $10^{-18}$ neper $/ \mathrm{cm}$ at $100 \mathrm{kHz}$, which is neglible compared with the total absorption. In fact, the absorption in Figures 6 and 7 does not exhibit so strong a dependence on frequency as might be expected to be proportional to $\omega^{4}$ from eq 2 .

The second origin of the absorption in suspensions is the viscous drag loss $\alpha_{v}$, which is 
given $^{12}$ under the condition $k a \ll 1$ through the relation

$$
\frac{\alpha_{\mathrm{v}}}{c}=\frac{k}{2} \frac{\frac{9}{4 z}\left(1+\frac{1}{z}\right)(\sigma-1)}{\left\{\frac{9}{42}\left(1+\frac{1}{z}\right)\right\}^{2}+\left\{\frac{2 \sigma-1}{2}+\frac{9 z}{4}\right\}^{2}}
$$

where $z=\beta_{1} a$ and $\beta_{1}=\left(\omega \rho_{1} / 2 \eta_{1}\right)^{1 / 2}$ is the propagation constant of viscous shear wave in the medium. The value of $\alpha_{\mathrm{v}} / c$ estimated from eq 3 is $10^{-6}$ neper $/ \mathrm{cm}$ at $100 \mathrm{kHz}$, being much smaller than the observed absorption coefficient.

The loss due to heat transfer between particle and medium is written under the condition of $k a \ll 1$ as $^{7}$

$$
\begin{aligned}
\frac{\alpha_{t}}{c} & =\text { Real part }\left\{\frac{3 T \rho_{1} u_{1}}{2 a^{2}}\left(\frac{\theta_{2}}{\rho_{2} C_{2}}-\frac{\theta_{1}}{\rho_{1} C_{1}}\right)\right. \\
& \left.\times \frac{D_{1} D_{2}[(1+i) x+1][(1+i) y-t h(1+i) y]}{D_{2}[(1+i) y-\operatorname{th}(1+i) y]+D_{1}[(1+i) x+1] \operatorname{th}(1+i) y}\right\}
\end{aligned}
$$

where $T$ is the absolute temperature, $C$ the specific heat at constant pressure, $D$ the thermal conductivity, $\theta$ the thermal expansion coefficient, $x=\gamma_{1} a$ and $y=\gamma_{2} a$ in which $\gamma$ is the propagation constant of thermal wave, $\gamma=(\omega \rho C / 2 D)^{1 / 2}$, and $i=\sqrt{-1}$. As proved from eq $4 \alpha_{t}$ is proportional to $\omega^{2}$ at low frequency limit where $y \ll 1$ but to $\omega^{1 / 2}$ at high frequency limit where $y \gg 1$. The value of $\alpha_{t} / c$ is calculated as $10^{-5}$ neper $/ \mathrm{cm}$ at $100 \mathrm{kHz}$ from eq 4 using values of thermal quantities of SBR at $20^{\circ} \mathrm{C}$. It can be safely concluded $\alpha_{t}$ is negligible compared with the observed absorption $\left(\alpha-\alpha_{1}\right)$ even at the high frequency limit of the present study.

Accordingly, it is assumed here that, over the covered frequency range, the excess absorption is ascribed to the internal loss in the particle which is given by ${ }^{12}$

$$
\frac{\alpha_{i}}{c}=\left(\frac{k}{2}\right) \tau\left(\frac{K^{\prime \prime}}{K^{\prime}}\right)
$$

where $K^{\prime}$ and $K^{\prime \prime}$ are real and imaginary parts of bulk modulus of the particle (the suffix 2 is omitted in the following).

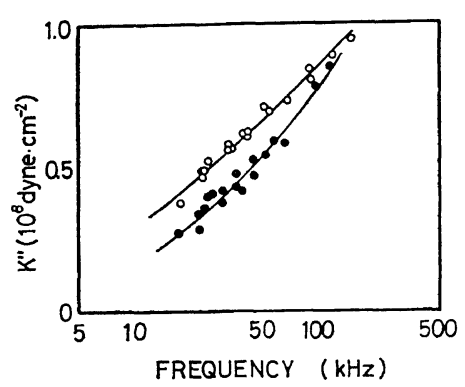

Figure 8. Loss part of complex bulk modulus of SBR for samples D (open circle) and E (filled circle).
Figure 8 shows $K^{\prime \prime}$ calculated from eq 5 . In this calculation, the $K^{\prime}$ value was taken from the data by Wada. ${ }^{12}$ The loss modulus $K^{\prime \prime}$ increases with increasing frequency because the range is the low frequency side of the primary relaxation due to segmental motion in the amorphous polymer above the glass temperature. The value is somewhat smaller for sample $E$ than D.

Wada, et al. ${ }^{12}$ measured the temperature dependence of absorption of ultrasonic waves in suspensions of SBR at $1 / 3,1$, and $3 \mathrm{MHz}$, and calculated $K^{\prime \prime}$ following the same procedure as above. They obtained the master curve at a reference temperature $T_{s}, 20^{\circ} \mathrm{C}$, assuming the frequency-temperature reducibility with the shift factor by Williams, Landel, and Ferry, ${ }^{13}$

$$
\log a_{T}=-\frac{8.86\left(T-T_{s}\right)}{101.6+\left(T-T_{s}\right)}
$$

The master curve thus obtained covers the frequency range $3 \mathrm{kHz}$ to $3 \times 10^{2} \mathrm{MHz}$. The value of $K^{\prime \prime}$ at 10 to $100 \mathrm{kHz}$ in the master curve is considerably larger than the value of Figure 8 in the present work.

The difference in $K^{\prime \prime}$ in the two samples in the present work and the one in Wada's report reflects the difference in the glass temperature. Since the sample used by Wada has a high glass temperature $\left(-25^{\circ} \mathrm{C}\right)$ on account of its high styrene content, the loss peak frequency of the primary relaxation is positioned at a low frequency and hence $K^{\prime \prime}$ at the low frequency side of the relaxation is high. The sample $E$ includes a larger fraction of low molecular weight com- 
ponent than $\mathrm{D}$, and hence its glass temperature is expected to be lower than sample $\mathrm{D}$, resulting in lower values of $K^{\prime \prime}$.

\section{CONCLUSION}

The resonant reverberation method which was first developed by the present authors for measuring acoustic absorption of liquids at frequencies as low as 10 to $300 \mathrm{kHz}$ was applied to polymer solutions and polymer suspensions. The data of absorption in dilute solutions of polystyrene revealed, together with those at higher frequencies, the wide distribution of relaxation times in dynamic volume viscosity which seems to come from crank-shaft motion of polymer chains. Acoustic measurements over a wide frequency range may afford a new tool for the study of micro-Brownian motion of polymers in a dilute solution.

The absorption in SBR suspensions reflects the dynamic mechanical loss of the polymer particle and the present technique was proved to be useful for viscoelastic measurement of polymer particles in their as-polymerized state.

Acknowlegment. The authors wish to express thanks to Mr. O. Yano and Miss A. Tsukazaki for preparing the manuscript.

\section{REFERENCES}

1. T. Ohsawa and Y. Wada, Japan. J. Appl. Phys., 6, 1351 (1967).

2. T. Osawa and Y. Wada, Japan. J. Appl. Phys., 8, 411 (1969).

3. J. D. Ferry, "Viscoelastic Properties of Polymers", John Wiley, New York, (1970).

4. P. E. Rouse, Jr., J. Chem. Phys., 21, 1272 (1953).

5. B. H. Zimm, J. Chem. Phys., 24, 266 (1956).

6. Y. Wada, H. Hirose, H. Umebayashi, and M. Otomo, J. Ppys. Soc. Japan, 15, 2324 (1960).

7. M. A. Isakovich, Zh. Eksper. Teor. Phys., 10 907 (1948).

8. K. Okano, "Solid State Physics," Vol. 14 Academic Press, New York, 1963, p 407.

9. H. Hässler and H.-J. Bauer, Kolloid-Z., 230, 194 (1969).

10. R. Cerf, R. Zana, and J. Candau, C. R. Acad. Sci. Paris, 254, 1061 (1962).

11. H. Nomura, S. Kato, and Y. Miyahara, Nippon Kagaku Zassi (J. Chem. Soc. Japan, Pure Chem. Sect.), 89, 149 (1968).

12. Y. Wada, R. Ito, and H. Ochiai, J. Phys. Soc. Japan, 17, 213 (1962).

13. M. L. Williams, R. F. Landel, and J. D. Ferry, J. Amer. Chem. Soc., 77, 3701 (1955). 